\title{
Liver Transplantation for Hepatocellular Carcinoma. Working Group Report from the ILTS Transplant Oncology Consensus Conference
}

Neil Mehta, MD, ${ }^{1}$ Prashant Bhangui, MBBS, MS, ${ }^{2}$ Francis Y. Yao, MD, ${ }^{1,3}$ Vincenzo Mazzaferro, MD, ${ }^{4}$ Christian Toso, MD, PhD, ${ }^{5}$ Nobuhisa Akamatsu, MD, PhD, ${ }^{6}$ Francois Durand, MD, ${ }^{7}$ Jan ljzermans, MD, PhD, ${ }^{8}$ Wojciech Polak, MD, PhD, ${ }^{8}$ Shusen Zheng, MD, PhD, ${ }^{9}$ John P. Roberts, MD, ${ }^{3}$ Gonzalo Sapisochin, MD, PhD, ${ }^{10}$ Taizo Hibi, MD, PhD, ${ }^{11}$ Nancy Man Kwan, MD, PhD, ${ }^{12}$ Mark Ghobrial, MD, PhD, ${ }^{13}$ and Avi Soin, MD ${ }^{2}$

\begin{abstract}
Liver transplantation (LT) offers excellent long-term outcome for certain patients with hepatocellular carcinoma (HCC), with a push to not simply rely on tumor size and number. Selection criteria should also consider tumor biology (including alpha-fetoprotein), probability of waitlist and post-LT survival (ie, transplant benefit), organ availability, and waitlist composition. These criteria may be expanded for live donor LT (LDLT) compared to deceased donor LT though this should not adversely affect the double equipoise in LDLT, namely ensuring both acceptable recipient outcomes and donor safety. HCC patients with compensated liver disease and minimal tumor burden have low urgency for $L T$, especially after local-regional therapy with complete response, and do not appear to derive the same benefit from LT as other waitlist candidates. These guidelines were developed to assist in selecting appropriate HCC patients for both deceased donor LT and LDLT.
\end{abstract}

(Transplantation 2020;104: 1136-1142).

\section{INTRODUCTION}

Liver transplantation (LT) offers excellent long-term outcome for certain patients with hepatocellular carcinoma (HCC), with a recent push to incorporate markers of tumor biology into selection criteria, rather than simply focusing on tumor size and number. In this working group report, we discuss how selection criteria and acceptable posttransplant outcomes for HCC patients undergoing living donor LT (LDLT) versus deceased donor LT (DDLT) should be different along with the optimal surgical management of patients presenting with a solitary small HCC. The aim of this guideline, approved by the International Liver Transplantation Society, is to provide a collection of expert opinions, consensus, and best practices surrounding

Received 4 December 2019.

Accepted 13 January 2020.

${ }^{1}$ Department of Medicine, University of California San Francisco, San Francisco, CA. ${ }^{2}$ Institute of Liver Transplantation and Regenerative Medicine, Medanta, The Medicity, Gurgaon, India.

${ }^{3}$ Department of Surgery, University of California San Francisco, San Francisco, CA.

${ }^{4}$ Department of Surgery, GI Surgery and Liver Transplantation, Istituto Nazionale Tumori, Milan, Italy.

${ }^{5}$ Division of Abdominal Surgery, University Hospitals of Geneva, Geneva, Switzerland.

${ }^{6}$ Department of Surgery, The University of Tokyo, Tokyo, Japan.

${ }^{7}$ Service d'Hépatologie \& Réanimation Hépatodigestive, Université Paris VII Hôpital Beaujon, Paris, France.

${ }^{8}$ Department of Surgery, Erasmus University Medical Center, Rotterdam, The Netherlands.

${ }^{9}$ Department of Surgery, First Affiliated Hospital, Zhejiang University, Hangzhou, China.
LT for HCC. Intended for use by physicians, these recommendations support specific approaches to the appropriate use of both deceased donor and live donor LT in the management of patients with HCC.

\section{DECEASED DONOR LT FOR HCC}

\section{Selection Criteria}

LT remains the optimal treatment strategy for patients with early-stage HCC. LT is thought to be the best oncologic resection, replaces the diseased liver, and restores normal hepatic function. For patients with HCC exceeding the Milan criteria ${ }^{1}$ ( 1 lesion $\leq 5 \mathrm{~cm}$ or $2-3$ lesions $\leq 3 \mathrm{~cm}$ ), survival after LT incrementally decreases with increasing

\footnotetext{
${ }^{10}$ Multi-Organ Transplant and HPB Surgical Oncology, Toronto General Hospital, University of Toronto, Toronto, Canada.

${ }^{11}$ Kumamoto University Graduate School of Medical Sciences, Kumamoto, Japan.

${ }^{12}$ Department of Surgery, LKS Faculty of Medicine, The University of Hong Kong, Hong Kong SAR, China.
}

${ }^{13}$ J C Walter Jr Transplant Center, Sherrie and Alan Conover Center for Liver Disease and Transplantation, Weill Cornell Medical College, Houston Methodist Hospital and Research Institute, Houston, TX.

The authors declare no funding or conflicts of interest.

All authors contributed equally to this manuscript as part of a working group of the ILTS consensus conference held in Rotterdam in February 2019.

Correspondence: Neil Mehta, MD, University of California, San Francisco, 513 Parnassus Ave, Room S-357, San Francisco, CA 94143-0538. (neil.mehta@ ucsf.edu).

Copyright (0) 2020 Wolters Kluwer Health, Inc. All rights reserved.

ISSN: 0041-1337/20/1046-1136

DOI: 10.1097/TP.0000000000003174 
tumor size and number ${ }^{2}$ although modest expansion of tumor size criteria to increase access to LT can achieve post-LT survival comparable to the Milan criteria. ${ }^{3-10}$

Especially in areas with organ shortages, the pendulum has largely swung away from expanded criteria for several reasons. These include an emphasis on performing LT in those HCC patients most likely to derive significant transplant survival benefit, ${ }^{11}$ concerns that implementation of expanded tumor criteria could reduce access to LT for patients with better post-LT prognosis, ${ }^{12,13}$ and longer wait times leading to increased use of local-regional therapy (LRT) as a bridge to $\mathrm{LT}^{14}$ There is also mounting evidence that tumor burden is just one of many factors that predict post-LT outcome. ${ }^{15,16}$ In this context, selection criteria have begun to shift to include surrogates of tumor biology (eg, alpha-fetoprotein [AFP]) and response to LRT, with a number of pre-LT selection criteria recently proposed. ${ }^{2,17-22}$

\section{Recommendations}

1. Indications for LT in HCC are aimed to cure cancer and improve patient's survival and quality of life (quality of evidence: moderate; strength of recommendation: strong).

2. Selection criteria should consider tumor biology (including AFP), tumor size and number, probability of survival, transplant benefit, organ availability, waitlist composition, and allocation priorities (quality of evidence: low; strength of recommendation: strong).

3. LT is recommended as a first-line option for HCC within Milan criteria, unsuitable for low-morbidity resection and ablation (quality of evidence: moderate; strength of recommendation: strong).

4. Consensus on expanded criteria for LT in HCC has not been reached but composite criteria that consider surrogates of tumor biology and response to neoadjuvant treatments, are likely to replace conventional morphological criteria for defining transplant feasibility (quality of evidence: moderate; strength of recommendation: strong).

\section{PREDICTION MODELS FOR DDLT}

Most pre-LT prediction models have incorporated serum makers in addition to various tumor burden cutoffs to more accurately determine recipient benefit. Post-LT survival begins to decline at an AFP of $\sim 20 \mathrm{ng} / \mathrm{mL}^{23,24}$ with worse survival as AFP increases. Therefore, various cutoffs have been utilized for exclusion from LT including $>400^{17}$ and $>1000 \mathrm{ng} / \mathrm{mL}$ in the United States. ${ }^{18}$ Patients with an elevated AFP who have a biochemical response to LRT have significantly better post-LT outcome than AFP nonresponders. ${ }^{25,26}$ Additional serum marker cutoffs associated with inferior post-LT outcome include neutrophil-to-lymphocyte ratio $>5$, AFP-L3 $>35 \%$, and des- $\gamma$ carboxyprothrombin $>7.5 \mathrm{ng} / \mathrm{mL}^{19,27,28}$ though these findings have not yet been validated. Several prediction models that allow for LT in HCC patients beyond Milan criteria require tumor biopsy and exclude patients with poorly differentiated tumor grade. With this approach, studies from Padova, ${ }^{29}$ Toronto, $^{21}$ and Hangzhou ${ }^{30}$ have shown acceptable postLT survival in HCC patients beyond Milan criteria. One caveat is that the overall agreement of preoperative needle core biopsy with explant histopathology is relatively poor. $^{31,32}$

\section{Recommendations}

1. Prognostication of post-LT outcome in patients with HCC, especially those beyond Milan criteria, should be based on measurable pre-LT conditions. Accuracy should be assessed in external independent prospective cohorts (quality of evidence: moderate; strength of recommendation: strong).

\section{DOWNSTAGING/BRIDGING TREATMENTS FOR DDLT}

LRT is frequently used with the aim of controlling tumor growth and reducing the risk of waitlist dropout, thus serving as a "bridge" to LT. While evidence supporting these bridging treatments in reducing waitlist dropout is limited, this approach seems justified when the waiting time for LT is expected to be at least 6 months. ${ }^{13}$ Those who exhibit tumor progression despite LRT have significantly worse post-LT outcomes when compared to those who demonstrate treatment response or stable disease following LRT. ${ }^{15,33-35}$ Observing tumor behavior over time after LRT may therefore allow for a more refined selection of candidates for $\mathrm{LT}^{36,37}$

The goal of downstaging is to reduce tumor size so that the residual viable tumors fall within acceptable LT criteria with most published studies using the Milan criteria as the endpoint of downstaging. ${ }^{38,39}$ The principle behind downstaging is to serve as a selection tool for a subset of patients with HCC beyond conventional LT criteria who would respond to downstaging treatments and do well after LT. This rationale is supported by the observation that post-LT outcomes in those successfully downstaged to Milan criteria are not significantly different from those who meet Milan criteria at presentation. ${ }^{40,41}$ There is also correlation between successful downstaging and a low prevalence of unfavorable explant histologic characteristics. ${ }^{40,42}$ In the United States, in an effort to standardize criteria for downstaging, the University of California, San Francisco downstaging protocol $^{40}$ has recently been adopted as a national policy for granting priority listing for LT. The initial selection criteria are single lesion $\leq 8 \mathrm{~cm}$, or $2-3$ lesions $<5 \mathrm{~cm}$ with total tumor diameter $<8 \mathrm{~cm}$, or $4-5$ nodules all $<3 \mathrm{~cm}$ with total tumor diameter $<8 \mathrm{~cm}$. The application of downstaging also involves a minimum observation period of 3 months of disease stability from successful downstaging to $\mathrm{LT}^{38,40}$

The use of more liberal inclusion criteria may result in a lower rate of successful downstaging and a higher rate of waitlist dropout, ${ }^{43,44}$ as well as inferior post-LT survival. ${ }^{45}$ In a recent analysis using the United Network for Organ Sharing database, ${ }^{45}$ Mehta et al observed similar 3-year post-LT survival among patients with HCC always within Milan criteria $(83 \%)$ compared to the group successfully downstaged using the above inclusion criteria (79\%). In contrast, the 3-year post-LT survival was significantly lower at $71 \%$ in the "all-comers" downstaging group with initial tumor burden beyond these criteria.

There are obviously safety concerns related to downstaging, including hepatic decompensation following LRT. It has been proposed that only patients with adequate hepatic function (Child's A/B, bilirubin $\leq 3 \mathrm{mg} / \mathrm{dL}$ ) should undergo downstaging, ${ }^{38}$ based on recommended guidelines for transarterial chemoembolization. ${ }^{46}$ Transarterial chemoembolization is the most commonly used treatment 
modality in published series ${ }^{38,39}$ and remains the recommended first-line treatment for downstaging. Y-90 radioembolization has shown promise as a downstaging treatment but requires further study. ${ }^{47}$

\section{Recommendations}

1. Although selection bias is likely, patients with HCC listed for LT receiving bridging therapies with objective response demonstrate improved waitlist and posttransplant outcomes (quality of evidence: moderate; strength of recommendation: strong).

2. Patients beyond Milan criteria can be considered for LT after successful downstaging, within defined protocols (quality of evidence: moderate; strength of recommendation: strong).

3. Tumor burden and tumor biology are good predictors of successful downstaging of HCC. Eligibility to downstaging should be defined upfront. In case of response, a notreatment period to assess end-treatment sustainability is recommended (quality of evidence: moderate; strength of recommendation: strong).

4. The degree of tumor response to bridging treatment may help in defining LT priority in patients listed with HCC (quality of evidence: moderate; strength of recommendation: strong).

\section{LIVE DONOR LT FOR HCC}

\section{Selection Criteria/prediction Models for LDLT}

Guided by strong recent evidence, the majority of centers now use a combination of morphological (eg, Milan, ${ }^{1}$ UCSF criteria ${ }^{3}$ ) and biological criteria to allocate livers for DDLT in patients with HCC, to select those that will benefit most from LT. However, in the "no competition" situation with LDLT, the ethical as well as scientific grounds have shifted. Several expanded criteria for LDLT have been proposed, although none have been externally validated..$^{30,48-54}$ The question that still remains is how far LT criteria can be expanded without adversely affecting the double equipoise in LDLT, namely ensuring both acceptable recipient outcomes and donor safety. ${ }^{55}$

Some predictive models incorporating tumor biology in selection criteria combining tumor burden, biomarkers, and ${ }^{18}$ F-FDG PET avidity have been reported in the LDLT setting, including the National Cancer Center Korea and Japanese criteria. ${ }^{28,56-60}$ One interesting aspect regarding selection criteria is that centers with longer wait times use downstaging/response to LRT by necessity whereas centers which primarily perform LDLT and have short wait times tend to have fairly liberal tumor burden criteria but rely heavily on biomarkers and/or negative ${ }^{18} \mathrm{~F}$-fluorodeoxyglucose positron emission tomography scan. FDG-negative patients beyond Milan criteria have satisfactory post-LT outcome ${ }^{56,58-60}$ whereas those with a tumor to nontumor ratio $>2$ tend to do poorly. In the future, molecular criteria (eg, angiotensin 2, VEGF, miR-718, pERK, glycipan 3, osteopontin) may take center stage. ${ }^{61,62}$

\section{Recommendations}

1. Selection criteria for patients with HCC may be different in LDLT than DDLT in selected cases (quality of evidence: moderate; strength of recommendation: strong).
2. Selection of patients outside standard criteria for LDLT may use validated criteria based on AFP and DCP cutoffs (eg, $<400$ and $<7.5 \mathrm{ng} / \mathrm{mL}$, respectively), ${ }^{18}$ F-FDG PET nonavid tumor, and if applicable, response to LRT to ensure acceptable tumor biology. They should have no extra hepatic disease and/or macrovascular invasion (quality of evidence: moderate; strength of recommendation: strong).

\section{Defining Minimal Survival Benefit Combined With Donor Risk}

Attempting to maximize recipient benefit while minimizing donor risk reflects the basic tenet of achieving double equipoise. ${ }^{55,63,64}$ There are perceived risks of transplanting patients with HCC too quickly without a minimal period of observation for tumor progression, as illustrated in the "fast-tracking" and "ablate and wait" concepts. 36,65 Additionally, there has been concern that graft regeneration in LDLT could lead to tumor growth and recurrence. However, several series and a meta-analysis have shown that LDLT achieves comparable outcomes to DDLT in HCC, thus largely negating these concerns. ${ }^{66,67}$

Previous reports have proposed an acceptable minimum survival of $50 \%$ at 5 -year after LDLT for HCC. ${ }^{68}$ An International HCC Consensus Conference report from $2012^{13}$ suggested that expansion beyond Milan criteria should take into account the effect of delaying LT for all potential waitlist candidates, including the ones with nontumor indications. Hence, the resulting proposal was to reserve LDLT for HCC patients who have an expected survival comparable to that of non-HCC patients (ie, $70 \%-80 \%$ at 5 y). Using a Markov model, Volk et $\mathrm{al}^{12}$ showed that the adverse effects of expanding LT criteria would outweigh its benefits if the expected 5 -year overall survival of a patient transplanted outside Milan criteria was $<61 \%$. Taking each of these previous reports into consideration, we believe that a minimum 5 -year postLDLT survival of $60 \%$ is an acceptable benchmark.

The triple (triangular) equipoise concept elaborates on the balance of 3 ethical dimensions-donor safety, expected recipient outcome, and recipient need. ${ }^{69}$ The risk of death for a recipient with a LDLT option is approximately half that of a patient awaiting DDLT without live donor options ${ }^{70}$ and this risk falls even further if an HCC patient with an LDLT option has a MELD score $>15 .^{71}$ Hence, it seems clear that HCC patients benefit from and deserve LDLT, especially in areas where DDLT rates are low. ${ }^{72}$ Similar to the paradigm with DDLT, the "transplant benefit" principle ${ }^{73}$ is a key concept for LDLT. By prioritizing patients based on life-years gained with LT, the transplant benefit principle performs better than urgency and utility schemes from a population perspective. ${ }^{74}$

In terms of donor risk, a worldwide survey with 11553 liver donors reported a mortality of $0.2 \%$, transplant rate of $0.04 \%$ for donor liver failure, and an overall donor morbidity of $24 \%{ }^{75}$ An overall donor complication rate of $<27 \%$ with $<6 \%$ Clavien-Dindo grade $3 / 4$ complications has been considered acceptable in a benchmark study ${ }^{76}$ However, we believe that centers should aim for zero donor mortality with maximum acceptable live donor risk of $<20 \%$ for Clavien grade I/2 complications and $<5 \%$ for grade $3 / 4$ complications. ${ }^{77}$ 


\section{Recommendation}

1. Minimum acceptable recipient overall survival should be $60 \%$ at 5 years after LDLT (quality of evidence: moderate; strength of recommendation: strong).

2. The goal live donor risk should be a Clavien $1 / 2$ complication rate $<20 \%$ and $3 / 4$ rate $<5 \%$ aiming for zero donor mortality (quality of evidence: moderate; strength of recommendation: strong).

3. The donor should be informed about the recipient's prognosis based on established criteria, center results, and published evidence (quality of evidence: low; strength of recommendation: strong).

\section{Role of Tumor Downstaging in LDLT}

Whereas the "ablate and wait" policy is relevant in DDLT, its applicability for LDLT is questionable. Downstaging tumors from beyond to within conventional criteria has been demonstrated to improve outcomes in HCC patients following LT $^{39-42}$ though most downstaging studies have been primarily in the DDLT setting. Only approximately $60 \%$ of patients with HCC beyond conventional downstaging criteria (so-called "all-comers") can be successfully downstaged to Milan criteria with LRT. $^{43}$ Therefore, UCSF criteria may be a more achievable downstaging endpoint before LDLT. Although some case series have reported successful downstaging of HCC with portal vein tumor thrombus often using Y-90 radio-embolization, ${ }^{78-81}$ the small number of patients and limited follow up make it difficult to propose guidelines for LDLT in this population at present.

\section{Recommendations}

1. HCC patients with tumor size and number beyond their "local" criteria should be downstaged with LRT at least to within UCSF criteria with AFP $<500 \mathrm{ng} / \mathrm{mL}$ before LDLT. An observation period of at least 3 months after successful downstaging is suggested before LDLT (quality of evidence: moderate; strength of recommendation: strong).

\section{MANAGEMENT OF SINGLE, SMALL HCC}

\section{Liver Transplantation Versus Hepatic Resection}

Hepatectomy for early-stage HCC is increasingly being performed due to both increased HCC incidence and organ shortages, and offers 5-year survival rates up to $60 \%{ }^{82}$ There are no randomized control trials evaluating resection versus LT, leading to the ongoing debate of which is most appropriate for patients within Milan criteria and adequate liver function. Resection confers up to 10 -fold higher odds of recurrence compared to $\mathrm{LT}^{1,83,84}$ and underlying cirrhosis increases recurrence risk after resection compared to normal background liver. ${ }^{85}$ In patients without significant fibrosis, resection is universally advised as first-line treatment, but in patients with compensated cirrhosis, recommendations are mixed and vary by tumor size and number. ${ }^{86}$

In patients otherwise eligible for LT, postresection 5 -year recurrence-free survival is $40 \%-50 \%{ }^{87,88}$ While 10 -year overall survival is better with LT for resectable HCC, ${ }^{89}$ many studies have shown similar 5-year overall survival for resection compared to LT in patients with a single $<3 \mathrm{~cm}$ HCC. ${ }^{89,90}$ Patients with a single $<3 \mathrm{~cm}$ HCC have improved outcome compared to those with larger tumor burden regardless of resection versus LT. ${ }^{91-93}$ More recently, a large multinational study ${ }^{94}$ reported a $\sim 40 \%$ cure rate with resection (compared to $75 \%$ with LT) in patients with single $<3 \mathrm{~cm}$ HCC and MELD $<11$ with similar intention-to-treat survival given the expected $10 \%-$ $20 \%$ waitlist dropout rate in those awaiting LT.

The question of offering LT to resectable, Child's A patients with single small HCC takes on greater importance after Berry and Ioannou ${ }^{95}$ found that HCC patients derive a significantly lower survival benefit from LT than non-HCC patients. Further, several studies have shown that HCC patients with favorable tumor (single tumor $<3 \mathrm{~cm}$ and AFP $\leq 20 \mathrm{ng} / \mathrm{mL}$ ) and liver-related characteristics (Child's A cirrhosis and MELD-Na $<15)^{11,96,97}$ have reduced urgency for LT. Such patients who subsequently undergo LRT with complete radiographic response have a very low risk of waitlist dropout ${ }^{11,96,98}$ and thus exceedingly low LT urgency with decreased LT survival benefit. ${ }^{37}$ This is especially true for HCC patients unlikely to have liver disease progression (eg, due to effective antiviral treatment or alcohol abstinence) and thus no alternate indication for LT.

\section{Recommendations}

1. HCC patients with compensated liver disease and minimal tumor burden have a low risk of waitlist dropout and do not derive the same immediate benefit from LT as other waitlist candidates (quality of evidence: moderate; strength of recommendation: strong).

2. Particularly in areas of organ shortages, due to competition with patients with higher transplant benefit, deceased donor LT is recommended only as second line treatment in resectable patients with single $<3 \mathrm{~cm}$ HCC in case of tumor recurrence or liver failure after resection or ablation (quality of evidence: moderate; strength of recommendation: conditional)

3. Patients with well-compensated disease and single $<3 \mathrm{~cm}$ HCC with complete response to LRT have reduced the urgency for LT (quality of evidence: moderate; strength of recommendation: strong).

\section{Salvage Liver Transplantation}

For patients undergoing resection, the strategy of salvage LT (SLT), or performing LT after recurrence within conventional transplant criteria, appears effective in 50\%$60 \%{ }^{99,100}$ with intention-to-treat survival $>80 \%$ at up to 10 years in patients who either do not recur after resection or who undergo LT after recurrence. ${ }^{99}$ Importantly, having early-stage HCC at resection predicts success with the SLT strategy. ${ }^{99}$ Similarly, Lee et al ${ }^{101}$ found that initial disease within Milan, single tumor, and lack of lymphovascular invasion predicted decreased likelihood of postresection recurrence beyond Milan criteria. Patients with single $<3 \mathrm{~cm}$ HCC would be expected to only have a $10-30 \%$ chance of recurrence beyond Milan and would likely be candidates for SLT in case of recurrence. Additionally, a recent systematic review and meta-analysis ${ }^{102}$ found improved 5-year post-LT survival after SLT compared to primary LT and concluded that SLT may be a better treatment strategy for recurrent HCC in compensated 
patients initially eligible for resection. While unlikely to apply to most patients with a single, small HCC, proposals to perform LT after resection in patients with a high risk for recurrence (eg, microvascular invasion and/or previously undiagnosed satellite lesions) ${ }^{103,104}$ require further validation.

\section{Recommendations}

1. Patients with single $<3 \mathrm{~cm}$ HCC who undergo resection but have tumor recurrence are highly likely to be eligible for SLT (quality of evidence: moderate; strength of recommendation: strong).

2. SLT and primary LT appear to have equivalent outcomes from the time of LT (quality of evidence: moderate; strength of recommendation: strong).

\section{REFERENCES}

1. Mazzaferro V, Regalia E, Doci R, et al. Liver transplantation for the treatment of small hepatocellular carcinomas in patients with cirrhosis. N Engl J Med. 1996;334:693-699.

2. Mazzaferro V, Sposito C, Zhou J, et al. Metroticket 2.0 model for analysis of competing risks of death after liver transplantation for hepatocellular carcinoma. Gastroenterology. 2018;154:128-139.

3. Yao FY, Xiao L, Bass NM, et al. Liver transplantation for hepatocellular carcinoma: validation of the UCSF-expanded criteria based on preoperative imaging. Am J Transplant. 2007;7:2587-2596.

4. Herrero JI, Sangro B, Quiroga J, et al. Influence of tumor characteristics on the outcome of liver transplantation among patients with liver cirrhosis and hepatocellular carcinoma. Liver Transpl. 2001;7:631-636

5. Herrero Jl, Sangro B, Pardo F, et al. Liver transplantation in patients with hepatocellular carcinoma across milan criteria. Liver Transpl. 2008;14:272-278.

6. Roayaie S, Frischer JS, Emre SH, et al. Long-term results with multimodal adjuvant therapy and liver transplantation for the treatment of hepatocellular carcinomas larger than 5 centimeters. Ann Surg. 2002;235:533-539.

7. Kneteman NM, Oberholzer J, Al Saghier M, et al. Sirolimus-based immunosuppression for liver transplantation in the presence of extended criteria for hepatocellular carcinoma. Liver Transpl. 2004; 10:1301-1311.

8. Silva M, Moya A, Berenguer M, et al. Expanded criteria for liver transplantation in patients with cirrhosis and hepatocellular carcinoma. Liver Transpl. 2008;14:1449-1460.

9. Guiteau JJ, Cotton RT, Washburn WK, et al. An early regional experience with expansion of Milan criteria for liver transplant recipients. Am J Transplant. 2010;10:2092-2098.

10. Mazzaferro V, Llovet JM, Miceli R, et al; Metroticket Investigator Study Group. Predicting survival after liver transplantation in patients with hepatocellular carcinoma beyond the milan criteria: a retrospective, exploratory analysis. Lancet Oncol. 2009;10:35-43.

11. Lai Q, Vitale A, lesari S, et al; European Hepatocellular Cancer Liver Transplant Study Group. Intention-to-treat survival benefit of liver transplantation in patients with hepatocellular cancer. Hepatology. 2017;66:1910-1919

12. Volk ML, Vijan S, Marrero JA. A novel model measuring the harm of transplanting hepatocellular carcinoma exceeding Milan criteria. Am J Transplant. 2008;8:839-846.

13. Clavien PA, Lesurtel M, Bossuyt PM, et al; OLT for HCC Consensus Group. Recommendations for liver transplantation for hepatocellular carcinoma: an international consensus conference report. Lancet Oncol. 2012;13:e11-e22.

14. Lee MW, Raman SS, Asvadi NH, et al. Radiofrequency ablation of hepatocellular carcinoma as bridge therapy to liver transplantation: a 10-year intention-to-treat analysis. Hepatology. 2017;65:1979-1990.

15. Lai Q, Avolio AW, Graziadei I, et al; European Hepatocellular Cancer Liver Transplant Study Group. Alpha-fetoprotein and modified response evaluation criteria in solid tumors progression after locoregional therapy as predictors of hepatocellular cancer recurrence and death after transplantation. Liver Transpl. 2013;19:1108-1118.
16. Mehta N, Yao FY. Hepatocellular cancer as indication for liver transplantation: pushing beyond Milan. Curr Opin Organ Transplant. 2016;21:91-98.

17. Toso C, Meeberg G, Hernandez-Alejandro R, et al. Total tumor volume and alpha-fetoprotein for selection of transplant candidates with hepatocellular carcinoma: a prospective validation. Hepatology. 2015;62:158-165.

18. Hameed B, Mehta N, Sapisochin G, et al. Alpha-fetoprotein level > $1000 \mathrm{ng} / \mathrm{ml}$ as an exclusion criterion for liver transplantation in patients with hepatocellular carcinoma meeting the Milan criteria. Liver Transpl. 2014;20:945-951.

19. Halazun KJ, Najjar M, Abdelmessih RM, et al. Recurrence after liver transplantation for hepatocellular carcinoma: a new MORAL to the story. Ann Surg. 2017;265:557-564.

20. Duvoux C, Roudot-Thoraval F, Decaens T, et al; Liver Transplantation French Study Group. Liver transplantation for hepatocellular carcinoma: a model including $\alpha$-fetoprotein improves the performance of Milan criteria. Gastroenterology. 2012;143:986-94.e3; quiz e14.

21. Sapisochin G, Goldaracena N, Laurence JM, et al. The extended Toronto criteria for liver transplantation in patients with hepatocellular carcinoma: a prospective validation study. Hepatology. 2016;64:2077-2088.

22. Sasaki K, Firl DJ, Hashimoto K, et al. Development and validation of the HALT-HCC score to predict mortality in liver transplant recipients with hepatocellular carcinoma: a retrospective cohort analysis. Lancet Gastroenterol Hepatol. 2017;2:595-603.

23. Berry K, loannou GN. Serum alpha-fetoprotein level independently predicts posttransplant survival in patients with hepatocellular carcinoma. Liver Transpl. 2013;19:634-645.

24. Mehta N, Heimbach J, Harnois DM, et al. Validation of a Risk Estimation of Tumor Recurrence After Transplant (RETREAT) score for hepatocellular carcinoma recurrence after liver transplant. JAMA Oncol. 2017;3:493-500.

25. Halazun KJ, Tabrizian P, Najjar M, et al. Is it time to abandon the Milan criteria?: results of a bicoastal US collaboration to redefine hepatocellular carcinoma liver transplantation selection policies. Ann Surg. 2018:268:690-699.

26. Mehta N, Dodge JL, Roberts JP, et al. Alpha-fetoprotein decrease from $>1,000$ to $<500 \mathrm{ng} / \mathrm{ml}$ in patients with hepatocellular carcinoma leads to improved posttransplant outcomes. Hepatology. 2019;69:1193-1205

27. Chaiteerakij R, Zhang X, Addissie BD, et al. Combinations of biomarkers and Milan criteria for predicting hepatocellular carcinoma recurrence after liver transplantation. Liver Transpl. 2015;21:599-606.

28. Kaido T, Ogawa K, Mori A, et al. Usefulness of the Kyoto criteria as expanded selection criteria for liver transplantation for hepatocellular carcinoma. Surgery. 2013;154:1053-1060.

29. Cillo U, Vitale A, Grigoletto F, et al. Intention-to-treat analysis of liver transplantation in selected, aggressively treated HCC patients exceeding the Milan criteria. Am J Transplant. 2007;7:972-981.

30. Zheng SS, Xu X, Wu J, et al. Liver transplantation for hepatocellular carcinoma: Hangzhou experiences. Transplantation. 2008;85:1726-1732.

31. Pawlik TM, Delman KA, Vauthey JN, et al. Tumor size predicts vascular invasion and histologic grade: implications for selection of surgical treatment for hepatocellular carcinoma. Liver Transpl. 2005;11:1086-1092.

32. Court CM, Harlander-Locke MP, Markovic D, et al. Determination of hepatocellular carcinoma grade by needle biopsy is unreliable for liver transplant candidate selection. Liver Transpl. 2017;23:1123-1132.

33. Otto G, Herber S, Heise M, et al. Response to transarterial chemoembolization as a biological selection criterion for liver transplantation in hepatocellular carcinoma. Liver Transpl. 2006;12:1260-1267.

34. Millonig G, Graziadei IW, Freund MC, et al. Response to preoperative chemoembolization correlates with outcome after liver transplantation in patients with hepatocellular carcinoma. Liver Transpl. 2007;13:272-279.

35. Kim DJ, Clark PJ, Heimbach J, et al. Recurrence of hepatocellular carcinoma: importance of mRECIST response to chemoembolization and tumor size. Am J Transplant. 2014;14:1383-1390.

36. Roberts JP, Venook A, Kerlan R, et al. Hepatocellular carcinoma: ablate and wait versus rapid transplantation. Liver Transpl. 2010;16:925-929.

37. Mazzaferro V. Squaring the circle of selection and allocation in liver transplantation for HCC: an adaptive approach. Hepatology. 2016;63:1707-1717.

38. Yao FY, Fidelman N. Reassessing the boundaries of liver transplantation for hepatocellular carcinoma: where do we stand with tumor down-staging? Hepatology. 2016;63:1014-1025. 
39. Parikh ND, Waljee AK, Singal AG. Downstaging hepatocellular carcinoma: a systematic review and pooled analysis. Liver Transpl. 2015;21:1142-1152.

40. Yao FY, Mehta N, Flemming J, et al. Downstaging of hepatocellular cancer before liver transplant: long-term outcome compared to tumors within Milan criteria. Hepatology. 2015;61:1968-1977.

41. Ravaioli M, Grazi GL, Piscaglia F, et al. Liver transplantation for hepatocellular carcinoma: results of down-staging in patients initially outside the Milan selection criteria. Am J Transplant. 2008;8:2547-2557.

42. Mehta N, Guy J, Frenette CT, et al. Excellent outcomes of liver transplantation following down-staging of hepatocellular carcinoma to within Milan criteria: a multicenter study. Clin Gastroenterol Hepatol. 2018;16:955-964.

43. Sinha J, Mehta N, Dodge JL, et al. Are there upper limits in tumor burden for down-staging of hepatocellular carcinoma to liver transplant? Analysis of the all-comers protocol. Hepatology. 2019;70:1185-1196.

44. Murali AR, Romero-Marrero C, Miller C, et al. Predictors of successful downstaging of hepatocellular carcinoma outside Milan criteria. Transplantation. 2016;100:2391-2397.

45. Mehta N, Dodge JL, Grab JD, et al. National experience on downstaging of hepatocellular carcinoma before liver transplant: influence of initial tumor burden, alpha-fetoprotein, and wait time. Hepatology. 2020;71:943-954.

46. European Association for the Study of the Liver; European Organisation for Research and Treatment of Cancer. EASL-EORTC clinical practice guidelines: management of hepatocellular carcinoma. J Hepatol. 2012;56:908-943.

47. Lewandowski RJ, Kulik LM, Riaz A, et al. A comparative analysis of transarterial downstaging for hepatocellular carcinoma: chemoembolization versus radioembolization. Am J Transplant. 2009;9:1920-1928.

48. Sugawara Y, Tamura S, Makuuchi M. Living donor liver transplantation for hepatocellular carcinoma: Tokyo University series. Dig Dis. 2007;25:310-312.

49. Lee SG, Hwang S, Moon DB, et al. Expanded indication criteria of living donor liver transplantation for hepatocellular carcinoma at one large-volume center. Liver Transpl. 2008;14:935-945.

50. Ito T, Takada Y, Ueda M, et al. Expansion of selection criteria for patients with hepatocellular carcinoma in living donor liver transplantation. Liver Transpl. 2007;13:1637-1644.

51. Soejima $Y$, Taketomi A, Yoshizumi T, et al. Extended indication for living donor liver transplantation in patients with hepatocellular carcinoma. Transplantation. 2007;83:893-899.

52. Gondolesi GE, Roayaie S, Muñoz L, et al. Adult living donor liver transplantation for patients with hepatocellular carcinoma: extending UNOS priority criteria. Ann Surg. 2004;239:142-149.

53. Kaihara S, Kiuchi T, Ueda M, et al. Living-donor liver transplantation for hepatocellular carcinoma. Transplantation. 2003;75(3 Suppl):S37-S40.

54. Shimamura T, Akamatsu N, Fujiyoshi M, et al; Japanese Liver Transplantation Society. Expanded living-donor liver transplantation criteria for patients with hepatocellular carcinoma based on the Japanese Nationwide Survey: the 5-5-500 rule - a retrospective study. Transpl Int. 2019;32:356-368.

55. Pomfret EA, Lodge JP, Villamil FG, et al. Should we use living donor grafts for patients with hepatocellular carcinoma? Ethical considerations. Liver Transpl. 2011;17 (Suppl 2):S128-S132.

56. Lee SD, Lee B, Kim SH, et al. Proposal of new expanded selection criteria using total tumor size and (18)F-fluorodeoxyglucose - positron emission tomography/computed tomography for living donor liver transplantation in patients with hepatocellular carcinoma: the National Cancer Center Korea criteria. World J Transplant. 2016;6:411-422.

57. Lee JH, Cho Y, Kim HY, et al. Serum tumor markers provide refined prognostication in selecting liver transplantation candidate for hepatocellular carcinoma patients beyond the Milan criteria. Ann Surg. 2016;263:842-850.

58. Kang YK, Choi JY, Paeng JC, et al. Composite criteria using clinical and FDG PET/CT factors for predicting recurrence of hepatocellular carcinoma after living donor liver transplantation. Eur Radiol. 2019;29:6009-6017.

59. Kornberg A, Witt U, Schernhammer M, et al. Combining 18F-FDG positron emission tomography with up-to-seven criteria for selecting suitable liver transplant patients with advanced hepatocellular carcinoma. Sci Rep. 2017;7:14176.

60. Takada Y, Kaido T, Shirabe K, et al; LTX-PET study group of the Japanese Society of Hepato-Biliary-Pancreatic Surgery and the Japanese Liver Transplantation Society. Significance of preoperative fluorodeoxyglucose-positron emission tomography in prediction of tumor recurrence after liver transplantation for hepatocellular carcinoma patients: a Japanese multicenter study. J Hepatobiliary Pancreat Sci. 2017;24:49-57

61. Pommergaard HC, Burcharth J, Rosenberg J, et al. Serologic and molecular biomarkers for recurrence of hepatocellular carcinoma after liver transplantation: a systematic review and meta-analysis. Transplant Rev (Orlando). 2016;30:171-177.

62. De Stefano F, Chacon E, Turcios L, et al. Novel biomarkers in hepatocellular carcinoma. Dig Liver Dis. 2018;50:1115-1123.

63. Azoulay D, Bhangui P, Andreani P, et al. Short- and long-term donor morbidity in right lobe living donor liver transplantation: 91 consecutive cases in a European center. Am J Transplant. 2011;11:101-110.

64. Ghobrial RM, Freise CE, Trotter JF, et al; A2ALL Study Group. Donor morbidity after living donation for liver transplantation. Gastroenterology. 2008;135:468-476.

65. Kulik L, Abecassis M. Living donor liver transplantation for hepatocellular carcinoma. Gastroenterology. 2004;127(5 Suppl 1):S277-S282.

66. Bhangui P, Vibert E, Majno P, et al. Intention-to-treat analysis of liver transplantation for hepatocellular carcinoma: living versus deceased donor transplantation. Hepatology. 2011;53:1570-1579.

67. Liang W, Wu L, Ling X, et al. Living donor liver transplantation versus deceased donor liver transplantation for hepatocellular carcinoma: a meta-analysis. Liver Transpl. 2012;18:1226-1236.

68. Llovet JM, Burroughs A, Bruix J. Hepatocellular carcinoma. Lancet. 2003:362:1907-1917.

69. Miller CM, Smith ML, Diago Uso T. Living donor liver transplantation: ethical considerations. Mt Sinai J Med. 2012;79:214-222.

70. Wong TCL, Ng KKC, Fung JYY, et al. Long-term survival outcome between living donor and deceased donor liver transplant for hepatocellular carcinoma: intention-to-treat and propensity score matching analyses. Ann Surg Oncol. 2019;26:1454-1462.

71. Berg CL, Merion RM, Shearon TH, et al. Liver transplant recipient survival benefit with living donation in the model for endstage liver disease allocation era. Hepatology. 2011;54:1313-1321.

72. Roll GR, Parekh JR, Parker WF, et al. Left hepatectomy versus right hepatectomy for living donor liver transplantation: shifting the risk from the donor to the recipient. Liver Transpl. 2013;19:472-481.

73. Vitale A, Volk M, Cillo U. Transplant benefit for patients with hepatocellular carcinoma. World J Gastroenterol. 2013;19:9183-9188.

74. Schaubel DE, Guidinger MK, Biggins SW, et al. Survival benefitbased deceased-donor liver allocation. Am J Transplant. 2009;9(4 Pt 2):970-981.

75. Cheah YL, Simpson MA, Pomposelli JJ, et al. Incidence of death and potentially life-threatening near-miss events in living donor hepatic lobectomy: a world-wide survey. Liver Transpl. 2013;19:499-506.

76. Rössler F, Sapisochin G, Song G, et al. Defining benchmarks for major liver surgery: a multicenter analysis of 5202 living liver donors. Ann Surg. 2016;264:492-500.

77. Goja S, Yadav SK, Saigal S, et al. Right lobe donor hepatectomy: is it safe? A retrospective study. Transpl Int. 2018;31:600-609.

78. Jeong $\mathrm{Y}$, Shin MH, Yoon SM, et al. Liver transplantation after transarterial chemoembolization and radiotherapy for hepatocellular carcinoma with vascular invasion. J Gastrointest Surg. 2017;21:275-283.

79. Wigg A, Hon K, Mosel L, et al. Down-staging of hepatocellular carcinoma via external-beam radiotherapy with subsequent liver transplantation: a case report. Liver Transpl. 2013;19:1119-1124.

80. Han DH, Joo DJ, Kim MS, et al. Living donor liver transplantation for advanced hepatocellular carcinoma with portal vein tumor thrombosis after concurrent chemoradiation therapy. Yonsei Med J. 2016;57:1276-1281.

81. Choi HJ, Kim DG, Na GH, et al. The clinical outcomes of patients with portal vein tumor thrombi after living donor liver transplantation. Liver Transpl. 2017;23:1023-1031.

82. Fan ST, Mau Lo C, Poon RT, et al. Continuous improvement of survival outcomes of resection of hepatocellular carcinoma: a 20-year experience. Ann Surg. 2011;253:745-758.

83. Merchant N, David CS, Cunningham SC. Early hepatocellular carcinoma: transplantation versus resection: the case for liver resection. Int J Hepatol. 2011;2011:142085.

84. Menahem B, Lubrano J, Duvoux C, et al. Liver transplantation versus liver resection for hepatocellular carcinoma in intention to treat: an attempt to perform an ideal meta-analysis. Liver Transpl. 2017;23:836-844.

85. Sasaki K, Shindoh J, Margonis GA, et al. Effect of background liver cirrhosis on outcomes of hepatectomy for hepatocellular carcinoma. JAMA Surg. 2017;152:e165059. 
86. Manzini G, Henne-Bruns D, Porzsolt F, et al. Is there a standard for surgical therapy of hepatocellular carcinoma in healthy and cirrhotic liver? A comparison of eight guidelines. BMJ Open Gastroenterol. 2017;4:e000129.

87. Cha CH, Ruo L, Fong $Y$, et al. Resection of hepatocellular carcinoma in patients otherwise eligible for transplantation. Ann Surg. 2003;238:315-21; discussion 321.

88. Poon RT, Fan ST, Lo CM, et al. Long-term survival and pattern of recurrence after resection of small hepatocellular carcinoma in patients with preserved liver function: implications for a strategy of salvage transplantation. Ann Surg. 2002;235:373-382.

89. Vitale A, Cucchetti A, Qiao GL, et al. Is resectable hepatocellular carcinoma a contraindication to liver transplantation? A novel decision model based on "number of patients needed to transplant" as measure of transplant benefit. J Hepatol. 2014;60:1165-1171.

90. Li C, Zhu WJ, Wen TF, et al. Child-Pugh A hepatitis B-related cirrhotic patients with a single hepatocellular carcinoma up to $5 \mathrm{~cm}$ : liver transplantation vs. resection. J Gastrointest Surg. 2014;18:1469-1476.

91. Poon RT, Fan ST, Lo CM, et al. Difference in tumor invasiveness in cirrhotic patients with hepatocellular carcinoma fulfilling the Milan criteria treated by resection and transplantation: impact on long-term survival. Ann Surg. 2007;245:51-58.

92. Scatton O, Goumard C, Cauchy F, et al. Early and resectable HCC: definition and validation of a subgroup of patients who could avoid liver transplantation. J Surg Oncol. 2015;111:1007-1015.

93. Adam R, Bhangui P, Vibert E, et al. Resection or transplantation for early hepatocellular carcinoma in a cirrhotic liver: does size define the best oncological strategy? Ann Surg. 2012;256:883-891.

94. Pinna AD, Yang T, Mazzaferro V, et al. Liver transplantation and hepatic resection can achieve cure for hepatocellular carcinoma. Ann Surg. 2018;268:868-875.

95. Berry K, Ioannou GN. Comparison of liver transplant-related survival benefit in patients with versus without hepatocellular carcinoma in the United States. Gastroenterology. 2015;149:669-80; quiz e15.
96. Mehta N, Dodge JL, Goel A, et al. Identification of liver transplant candidates with hepatocellular carcinoma and a very low dropout risk: implications for the current organ allocation policy. Liver Transpl. 2013;19:1343-1353.

97. Mehta N, Dodge JL, Hirose R, et al. Predictors of low risk for dropout from the liver transplant waiting list for hepatocellular carcinoma in long wait time regions: implications for organ allocation. Am J Transplant. 2019;19:2210-2218.

98. Cucchetti A, Cescon M, Bigonzi E, et al. Priority of candidates with hepatocellular carcinoma awaiting liver transplantation can be reduced after successful bridge therapy. Liver Transpl. 2011;17:1344-1354.

99. de Haas RJ, Lim C, Bhangui P, et al. Curative salvage liver transplantation in patients with cirrhosis and hepatocellular carcinoma: an intention-to-treat analysis. Hepatology. 2018;67:204-215.

100. Bhangui $P$, Allard MA, Vibert E, et al. Salvage versus primary liver transplantation for early hepatocellular carcinoma: do both strategies yield similar outcomes? Ann Surg. 2016;264:155-163.

101. Lee SY, Konstantinidis IT, Eaton AA, et al. Predicting recurrence patterns after resection of hepatocellular cancer. HPB (Oxford). 2014;16:943-953

102. Yadav DK, Chen W, Bai X, et al. Salvage liver transplant versus primary liver transplant for patients with hepatocellular carcinoma. Ann Transplant. 2018;23:524-545.

103. Ferrer-Fàbrega J, Forner $\mathrm{A}$, Liccioni $\mathrm{A}$, et al. Prospective validation of ab initio liver transplantation in hepatocellular carcinoma upon detection of risk factors for recurrence after resection. Hepatology. 2016;63:839-849.

104. Tribillon E, Barbier L, Goumard C, et al. When should we propose liver transplant after resection of hepatocellular carcinoma? A comparison of salvage and de principe strategies. J Gastrointest Surg. 2016;20:66-76; discussion 76. 